\title{
Dieta, selectividad y solapamiento trófico entre las tallas del pez blanco Menidia humboldtiana (Atheriniformes: Atherinopsidae) en el embalse Tiacaque, México
}

\author{
Regina Sánchez, Abigahil Ochoa \& Angélica Mendoza \\ Facultad de Estudios Superiores Iztacala UNAM. Av. de los Barrios No. 1. Los Reyes Iztacala, Tlalnepantla, Edo. de \\ México, C.P. 54090. México; regmer14@yahoo.com.mx, danna_green@hotmail.com, efimerangie@yahoo.com.mx
}

\author{
Recibido 10-I-2012. Corregido 22-VIII-2012. Aceptado 21-IX-2012.
}

\begin{abstract}
Diet, selectivity and trophic overlap between the sizes of Silverside Menidia humboldtiana (Atheriniformes: Atherinopsidae) in the reservoir Tiacaque, Mexico. Menidia humboldtiana, a native species of Mexico, is a common inhabitant of local reservoirs. It represents a highly appreciated fish of economic importance in the central part of the country because of its delicate flavor. Trophic behavior of this species is important to understand the relationships with other fish species in reservoirs. With the aim to study this specific topic, the trophic spectrum, selectivity coefficient and overlap, were determined among different sizes of the Silverside $M$. humboldtiana. For this, both zooplankton and fish samples were taken during four different seasons of 1995. Zooplankton samples were taken through a mesh (125micron), and all organisms were identified to generic level. Fish were captured and grouped into standard length intervals per season, and the stomach contents were obtained and analyzed. Trophic interactions included the stomach contents analysis (Laevastu method), the coefficient of selection (Chesson) and the trophic overlap (Morisita index modified by Horn) between sizes. A total of 14 zooplankton genera were identified, of which Bosmina was the most abundant (29625 ind./10L) followed by Cyclops (9496 ind./10L), during the spring. Small size fishes (1-4.9cm) consumed high percentages of Cyclops in the spring (61.24\%) and winter (69.82\%). Ceriodaphnia was consumed by fish sizes of $3-10.9 \mathrm{~cm}(72.41 \%)$ and $13-14.9 \mathrm{~cm}(95.5 \%)$ during the summer; while in autumn, small sizes $(1-4.9 \mathrm{~cm})$ ingested Mastigodiaptomus and Ceriodaphnia; Daphnia and Bosmina were consumed by fishes of 5-8.9cm and the biggest sizes $(9-14.9 \mathrm{~cm})$ feed on Ceriodaphnia. M. humboldtiana makes a selective predation by the genera Ceriodaphnia, Daphnia, Mastigodiaptomus, Bosmina and Cyclops, depending on the size length interval. The trophic overlap was very marked among all sizes on spring, autumn and winter, unlike in summer fish of 1-2.9 and $11-12.9 \mathrm{~cm}$ did not show overlap with other length intervals. M. humboldtiana is a zooplanktivore species, which performs a selective predation and a marked trophic overlap between the different fish sizes. Rev. Biol. Trop. 61 (2): 787-796. Epub 2013 June 01.
\end{abstract}

Key words: food selectivity, Menidia humboldtiana, trophic overlap, silverside, zooplankton.

La ictiofauna mexicana dulceacuícola es rica en especies, esta riqueza está determinada entre otras causas por la gran diversidad de hábitats y por tener dentro de su territorio especies de la zona Neártica y de la zona Neotropical (Miller 2005). Menidia humboldtiana, conocido como pez blanco, es de importancia económica en la parte central del país, donde es muy apreciado por su delicado sabor.

La alimentación en los peces guarda gran importancia para el establecimiento de relaciones tróficas ligadas con la conservación. Las variaciones alimenticias y la selectividad que puedan ejercer los peces sobre su alimento, suelen relacionarse con alteraciones en las condiciones ambientales o con los requerimientos energéticos de los mismos e involucran cambios en el tamaño, tipo y cantidad de presas consumidas, siendo de gran importancia para comprender el comportamiento de las poblaciones del zooplancton en presencia de peces zooplanctófagos. 
Brooks \& Dodson (1965), Wells (1970) y Vanderploeg \& Scavia (1979), mencionan que al existir un gran número de peces zooplanctófagos en sistemas naturales, disminuyen sensiblemente las poblaciones del zooplancton de tallas grandes propiciando un aumento en el fitoplancton. Por el contrario, si la abundancia es baja, aumentan las poblaciones de zooplancton de tallas grandes disminuyendo con ello el fitoplancton. Se ha demostrado una selectividad de estos peces por una determinada talla de presa, guardando una estrecha relación entre el zooplancton de tamaño grande con la presencia de los mismos.

M. humboldtiana ha sido estudiada en su alimentación por Navarrete \& Cházaro (1992) quienes mencionan el consumo preferente de cladóceros, a diferencia Elías et al. (2008) refieren que consume de manera importante a los rotíferos (Keratella) y cladóceros (Bosmina). Yubi et al. (2008) y Navarrete et al. (2007) también registraron a cladóceros y copépodos (Mastigodiaptomus) como alimentos importantes. Sánchez et al. (2009) reportan que los peces consumen en altos porcentajes a los géneros Bosmina, Daphnia y Mastigodiaptomus.

La selectividad por el alimento comprende dos aspectos fundamentales, la amplitud total de la dieta del depredador y el consumo selectivo del depredador por determinada presa, al respecto Sánchez et al. (2009) reportan una depredación selectiva por los géneros Bosmina, Daphnia y Mastigodiaptomus. Por otra parte la amplitud del espectro trófico evalúa el grado de especialización trófica de un depredador y la selectividad por determinada presa está determinada por el tamaño, forma, color y movimiento de la presa.

Cuando dos clases de talla de un determinado pez tienen patrones similares de aprovechamiento de un recurso alimenticio, se dice que presentan un alto grado de solapamiento, lo que se traduce en una fuerte competencia intraespecífica. Cuando el solapamiento es pequeño se podría interpretar como una tendencia hacia la repartición de este recurso; al respecto Sánchez et al. (2009) estudiaron el grado de solapamiento para esta especie en el embalse Danxhó.

El conocimiento de estos aspectos nos permite entender el comportamiento de los peces nativos en los cuerpos de agua en los que se han realizado introducciones de peces exóticos, y que en muchos de los casos compiten por el alimento en alguna etapa de su vida (Navarrete \& Sánchez 1991). Se podrán plantear estrategias adecuadas para su cultivo, considerando el alimento vivo que se suministre en las diferentes etapas del desarrollo de los peces, de ahí que el objetivo de este trabajo fue determinar si el pez blanco M. humboldtiana selecciona su alimento, sobre qué géneros la realiza y establecer si existe solapamiento en la dieta entre las diferentes tallas del pez.

\section{MATERIALES Y MÉTODOS}

El presente estudio se realizó en el embalse Tiacaque, Estado de México, localizado entre $\operatorname{los} 19^{\circ} 40^{\prime} 16^{\prime \prime}-19^{\circ} 40^{\prime} 20^{\prime \prime} \mathrm{N}$ and $99^{\circ} 42^{\prime} 41^{\prime \prime}$ - 99 $42^{\prime} 15^{\prime \prime} \mathrm{W}$, a una altura de $2530 \mathrm{msnm}$ y con una extensión de $0.22 \mathrm{~km}^{2}$. El embalse pertenece a la Región Hidrológica Lerma-Chapala-Santiago, el clima de la zona de acuerdo a Köppen modificado por García (1973) es C $\left(\mathrm{w}_{2}\right)(\mathrm{w}) \mathrm{b}\left(\mathrm{i}^{\prime}\right)$ que corresponde al más húmedo de los templados subhúmedos.

Considerando las cuatro épocas del año 1995, se establecieron en el embalse tres estaciones de muestreo de acuerdo con las características morfométricas. De cada una, se obtuvo una muestra de zooplancton al filtrar 10L de agua del reservorio, a través de una red de $125 \mu \mathrm{m}$ de abertura de malla; estas muestras fueron seguidamente fijadas con formol al $4 \%$ (Gaviño et al. 1982). Los organismos en las muestras de zooplancton fueron determinados a nivel genérico mediante el uso de claves de Edmonson (1959), Pennak (1989) y Dussart \& Defaye (1995). Se registró el número de organismos por género en cada estación, por época del año.

En cada estación se obtuvo una muestra de peces mediante una red de chinchorro de $30 \mathrm{~m}$ de largo y $8 \mathrm{~mm}$ de luz de malla, los peces 
fueron fijados inmediatamente después de su captura con formol al 10\% para detener los procesos digestivos.

Para los análisis de los contenidos estomacales de los peces, éstos se agruparon en intervalos de longitud estándar para cada época del año $(1-2.9 \mathrm{~cm}, 3-4.9 \mathrm{~cm}, 5-6.9 \mathrm{~cm}, 7-8.9 \mathrm{~cm}$, $9-10.9 \mathrm{~cm}, 11-12.9 \mathrm{~cm}$ y $13-14.9 \mathrm{~cm})$. Los grupos zoológicos presentes en los contenidos se determinaron al nivel genérico con las claves antes mencionadas. El método utilizado para el análisis de los contenidos estomacales fue el volumétrico propuesto por Laevastu (1971). También se determinó el coeficiente de selección de acuerdo a Chesson (1978) que relaciona la proporción de la presa en la dieta, su contraparte en el ambiente y el número total de tipos de presa. Para evaluar el solapamiento trófico entre las tallas, se utilizó el índice de Morisita modificado por Horn (Arcifa et al. 1991).

\section{RESULTADOS}

Se registraron 14 géneros del zooplancton de los cuales Bosmina (Cladocera) fue el más abundante en primavera, verano e invierno (29625, 7349 y 6777 ind/10L, respectivamente). Seguido en orden de abundancia por Cyclops (Copepoda) de 3203 a 9496 ind/10L, este último en primavera (Cuadro 1). Ceriodaphnia (Cladocera) registró su máxima abundancia en otoño 7153 ind/10L, Daphnia (Cladocera) en primavera (1490 ind/10L) y Mastigodiaptomus (Copepoda) en otoño (2381 ind/10L). El rotífero Asplanchna fue abundante en primavera (9413 ind/10L).

Se analizaron un total de 348 peces durante las cuatro estaciones del año, con longitudes de $1-14.9 \mathrm{~cm}$, agrupándolos en siete intervalos de longitud. Los peces pequeños de $1-2.9 \mathrm{~cm}$ de longitud consumieron en altos porcentajes los géneros Cyclops 61.24 y $69.82 \%$ en primavera e invierno, respectivamente; Bosmina $72.83 \%$ en verano y Ceriodaphnia $48 \%$ en otoño (Fig. 1A). El intervalo de $3-4.9 \mathrm{~cm}$ consumieron en un $49.68 \%$ a Mastigodiaptomus en otoño, Cyclops en primavera e invierno (47.53 y $65.72 \%$, respectivamente), y en verano el género más consumido fue Ceriodaphnia (72.41\%) (Fig. 1B). A diferencia, para las tallas de $5-6.9 \mathrm{~cm}$, el cladócero más consumido fue Daphnia en primavera (76.95\%) y otoño

CUADRO 1

Densidad del zooplancton en el embalse Tiacaque

TABLE 1

Zooplankton density in the Tiacaque reservoir

\begin{tabular}{|c|c|c|c|c|}
\hline $\begin{array}{c}\text { Géneros } \\
\text { Zooplancton }\end{array}$ & $\begin{array}{c}\text { Primavera } \\
\text { (\# ind./10L) }\end{array}$ & $\begin{array}{c}\text { Verano } \\
\text { (\# ind./10L) }\end{array}$ & $\begin{array}{c}\text { Otoño } \\
\text { (\# ind./10L) }\end{array}$ & $\begin{array}{c}\text { Invierno } \\
\text { (\# ind./10L) }\end{array}$ \\
\hline Mastigodiaptomus & 678 & 62 & 2381 & 75 \\
\hline Cyclops & 9496 & 4777 & 6464 & 3203 \\
\hline Bosmina & 29625 & 7349 & 1287 & 6777 \\
\hline Daphnia & 1490 & 122 & 46 & 166 \\
\hline Ceriodaphnia & 1004 & 4835 & 7153 & 43 \\
\hline Alona & 164 & 33 & 4 & 76 \\
\hline Simocephalus & 76 & 0 & 0 & 119 \\
\hline Diaphanosoma & 19 & 99 & 23 & 20 \\
\hline Moina & 3 & 12 & 0 & 7 \\
\hline Macrothrix & 0 & 2 & 0 & 0 \\
\hline Asplanchna & 9413 & 147 & 519 & 1250 \\
\hline Brachionus & 0 & 17 & 0 & 25 \\
\hline Keratella & 15 & 1 & 2 & 82 \\
\hline Filinia & 0 & 0 & 0 & 153 \\
\hline
\end{tabular}



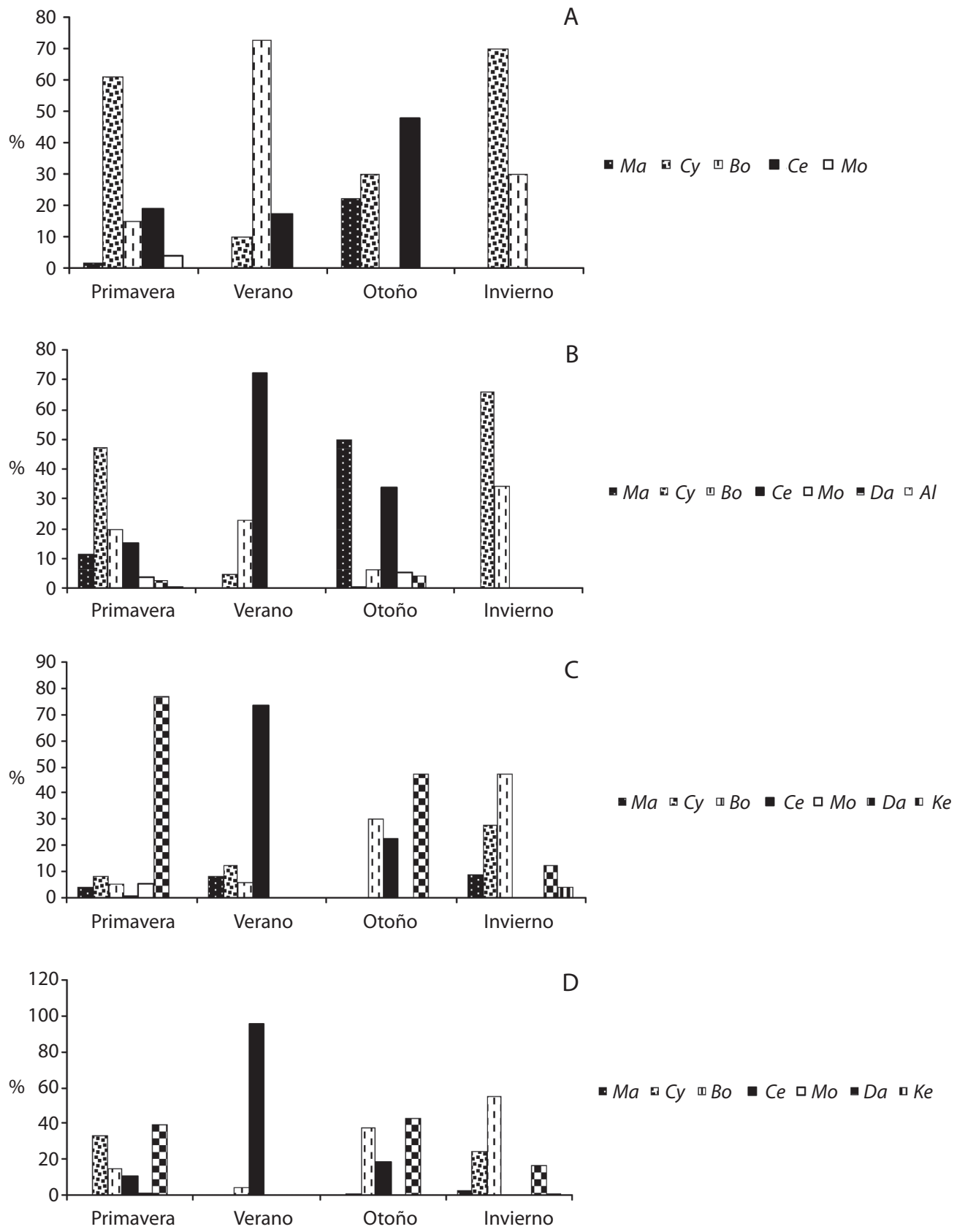

Fig. 1A-B. Espectro trófico de Menidia humboldtiana en el embalse Tiacaque. A. (1-2.9cm). B. (3-4.9cm). $\mathrm{Ma}=$ Mastigodiaptomus, $\mathrm{Cy}=$ Cyclops, $\mathrm{Bo}=$ Bosmina, $\mathrm{Ce}=$ Ceriodaphnia, $\mathrm{Mo}=$ Moina, $\mathrm{Da}=$ Daphnia, $\mathrm{Al}=$ Alona. Fig. 1C-D. Espectro trófico de Menidia humboldtiana en el embalse Tiacaque. C. (5-6.9cm). D. (7-8.9cm). Ma=Mastigodiaptomus, $\mathrm{Cy}=$ Cyclops, $\mathrm{Bo}=$ Bosmina, $\mathrm{Ce}=$ Ceriodaphnia, $\mathrm{Mo}=$ Moina, $\mathrm{Da}=$ Daphnia, $\mathrm{Ke}=$ Keratella .

Fig. 1A-B. Trophic spectrum of Menidia humboldtiana in the Tiacaque reservoir. A. $(1-2.9 \mathrm{~cm})$. B. (3-4.9cm). Fig. 1C-D. Trophic spectrum of Menidia humboldtiana in the Tiacaque reservoir. C. $(5-6.9 \mathrm{~cm})$. D. $(7-8.9 \mathrm{~cm})$. 
(47.16\%); sin embargo, varió en el verano con Ceriodaphnia (73.48\%) y Bosmina en invierno (47.51\%) (Fig. 1C). Para los peces de $7-8.9 \mathrm{~cm}$ los porcentajes más altos en los contenidos estomacales correspondieron a los cladóceros Ceriodaphnia, Bosmina y Daphnia
(95.5, 55.28 y $42.55 \%$, respectivamente) (Fig. 1D). Ceriodaphnia y Cyclops registraron altos porcentajes durante el verano y el otoño para el primero; primavera e invierno para el segundo (Fig. 1E), en los peces de $9-10.9 \mathrm{~cm}$. En el intervalo de $11-12.9 \mathrm{~cm}$ los géneros más importantes
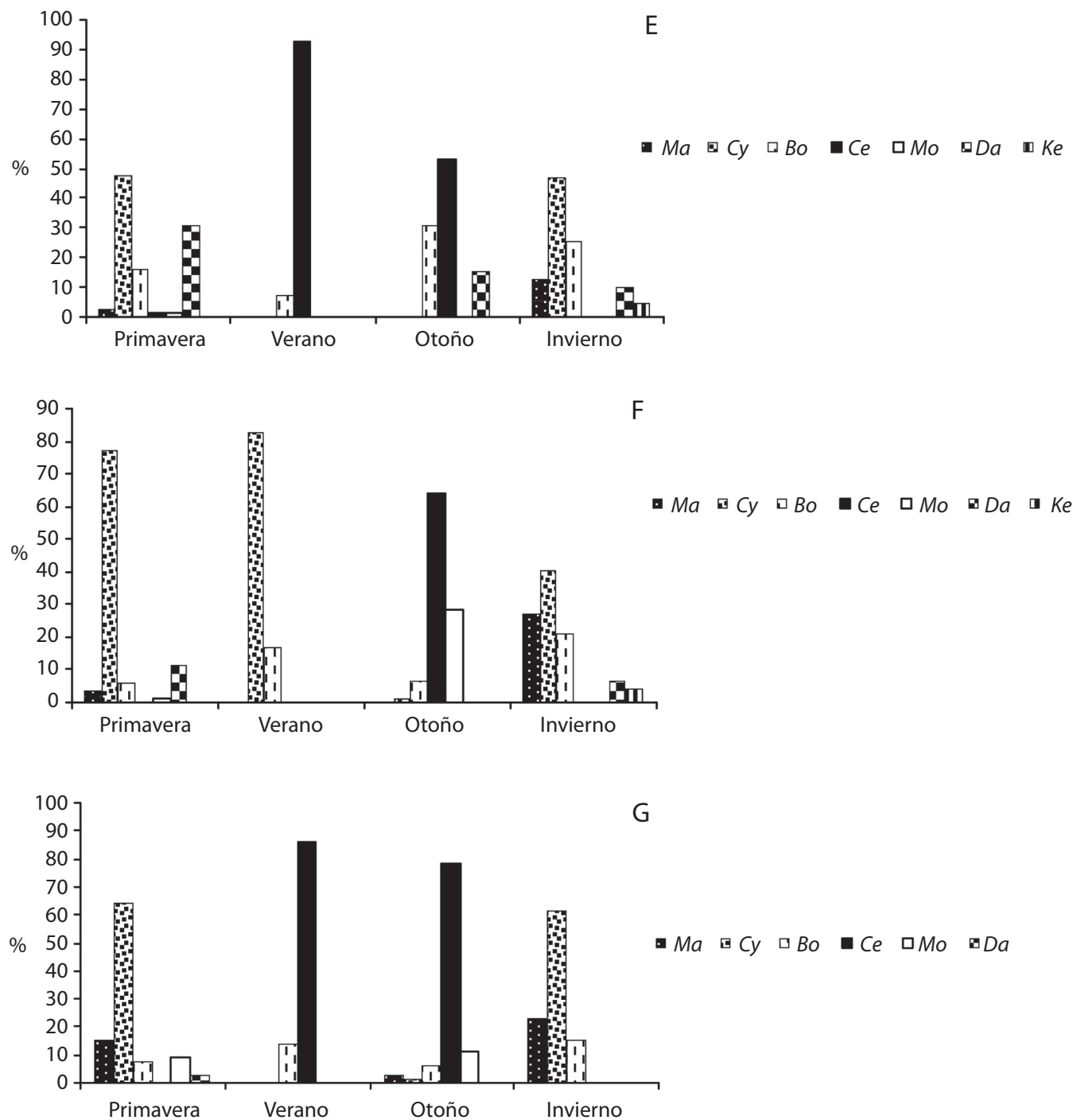

Fig. 1E-F. Espectro trófico de Menidia humboldtiana en el embalse Tiacaque. E. (9-10.9cm). F. (11-12.9cm). $\mathrm{Ma}=$ Mastigodiaptomus, $\mathrm{Cy}=$ Cyclops, $\mathrm{Bo}=$ Bosmina, $\mathrm{Ce}=$ Ceriodaphnia, Mo=Moina, Da=Daphnia, Ke=Keratella . Fig. 1G. Espectro trófico de Menidia humboldtiana $(13-14.9 \mathrm{~cm})$ en el embalse Tiacaque. $\mathrm{cm}) . \mathrm{Ma}=$ Mastigodiaptomus, Cy=Cyclops, $\mathrm{Bo}=$ Bosmina, $\mathrm{Ce}=$ Ceriodaphnia, $\mathrm{Mo}=$ Moina, $\mathrm{Da}=$ Daphnia .

Fig. 1E-F. Trophic spectrum of Menidia humboldtiana in the Tiacaque reservoir. E. (9-10.9cm). F. (11-12.9cm). Fig. 1G. Trophic spectrum of Menidia humboldtiana $(13-14.9 \mathrm{~cm})$ in the Tiacaque reservoir. 
fueron Cyclops en primavera (77.44\%), verano $(83.02 \%)$ e invierno $(40.48 \%)$ y Ceriodaphnia $(64.11 \%)$ en otoño (Fig. 1F). Finalmente, los peces más grandes también consumieron principalmente Cyclops y Ceriodaphnia (Fig. 1G).

Con respecto a los géneros seleccionados por Menidia humboldtiana se observó que en primavera las tallas pequeñas de $1-4.9 \mathrm{~cm}$ seleccionaron al género Ceriodaphnia, los peces de $5-12.9 \mathrm{~cm}$ a Daphnia y los más grandes a Mastigodiaptomus (Fig. 2A). A diferencia, en el verano los intervalos de tallas de $3-4.9 \mathrm{~cm}$ y de $7-14.9 \mathrm{~cm}$ seleccionaron a Ceriodaphnia y los más pequeños a Bosmina (Fig. 2B). En el otoño, el género Daphnia fue seleccionado por los peces de tallas entre $3-10.9 \mathrm{~cm}$, sin embargo, los peces más grandes $(11-14.9 \mathrm{~cm})$ seleccionaron a Ceriodaphnia y Bosmina (Fig. 2C). Para el invierno, la selectividad recayó sobre los copépodos Cyclops por las tallas pequeñas $(1-4.9 \mathrm{~cm})$, y Mastigodiaptomus, por los peces de $5-14.9 \mathrm{~cm}$ de longitud. Solo un género de cladóceros fue seleccionado, Daphnia por las tallas intermedias $(5-8.9 \mathrm{~cm})$ (Fig. 2D).

Solapamiento según tallas: En relación al solapamiento de la dieta entre los intervalos de longitud, en las cuatro épocas del año, se observó en primavera que los peces de $1-4.9 \mathrm{~cm}$ y de $7-14.9 \mathrm{~cm}$ dieron valores elevados de solapamiento (arriba de 0.9287, considerando que el valor de uno corresponde a un solapamiento total) y el intervalo de $5-6.9 \mathrm{~cm}$ quedó unido a este grupo con un valor de 0.7553 (Fig. 3). En verano, los peces pequeños $(1-2.9 \mathrm{~cm})$ y los más grandes $(11-12.9 \mathrm{~cm})$ se separaron de todos los demás intervalos. En otoño el solapamiento más elevado se dio con los intervalos de longitud de $5-8.9 \mathrm{~cm}$ y de $11-14.9 \mathrm{~cm}$ (con valores por arriba de 0.9559). En el invierno se observó un fuerte solapamiento entre las tallas pequeñas $(1-4.9 \mathrm{~cm})$ y las grandes $(9-14.9 \mathrm{~cm})$ con un valor de 0.9379 .

\section{DISCUSIÓN}

El pez blanco Menidia humboldtiana consumió ocho géneros del zooplancton de los
14 existentes, a diferencia esta misma especie consumió solo cuatro en el embalse Danxhó (Sánchez et al. 2009). Esto se puede deber, en primera instancia, a las abundancias del zooplancton en el ambiente, en general en Tiacaque se registraron las mayores abundancias para todos los géneros, si analizamos los principales grupos alimenticios, Bosmina registró abundancias de 1287-29625ind/10L en Tiacaque, a diferencia este mismo en Danxhó, cuyas abundancias oscilaron entre 69-2 247ind/10L; lo mismo ocurrió con Cyclops 3203-9496ind/10L y 113-292ind/10L en Tiacaque y Danxhó, respectivamente. Esto propició una mayor disponibilidad de alimento, lo que se tradujo en un mayor encuentro entre el depredador y su presa.

$\mathrm{Si}$ analizamos los espectros tróficos en relación con las abundancias de los grupos alimenticios en el ambiente, observamos que para primavera consumieron el mayor número de géneros zooplanctónicos (entre cinco y siete) por todos los intervalos de talla, así mismo se registraron en general las máximas abundancias para los géneros Cyclops, Bosmina, Daphnia y Alona. En el verano, solo ingirieron a Ceriodaphnia, Bosmina y Cyclops coincidiendo con abundancias elevadas en el ambiente, los demás géneros del zooplancton en esta época registraron sus menores abundancias. Para otoño e invierno aumentó el número de géneros ingeridos por los peces.

Con respecto a los diferentes intervalos de talla, los peces pequeños de $1-2.9 \mathrm{~cm}$, ingirieron preferentemente al género Cyclops en primavera e invierno, a Bosmina en verano y Ceriodaphnia en otoño, este último registró su mayor abundancia en el ambiente $(40.01 \%$ del zooplancton), es importante mencionar que en verano los peces de este intervalo sólo consumieron a Bosmina, Ceriodaphnia y Cyclops. Los peces de $3-4.9 \mathrm{~cm}$ ingirieron preferentemente a los copépodos Cyclops y Mastigodiaptomus en primavera y otoño respectivamente, coincidiendo con sus máximas abundancias (56.99\% y $13.32 \%$ del zooplancton).

Daphnia es el género que más aportó a la dieta de los peces de $5-6.9 \mathrm{~cm}$ en primavera 
A
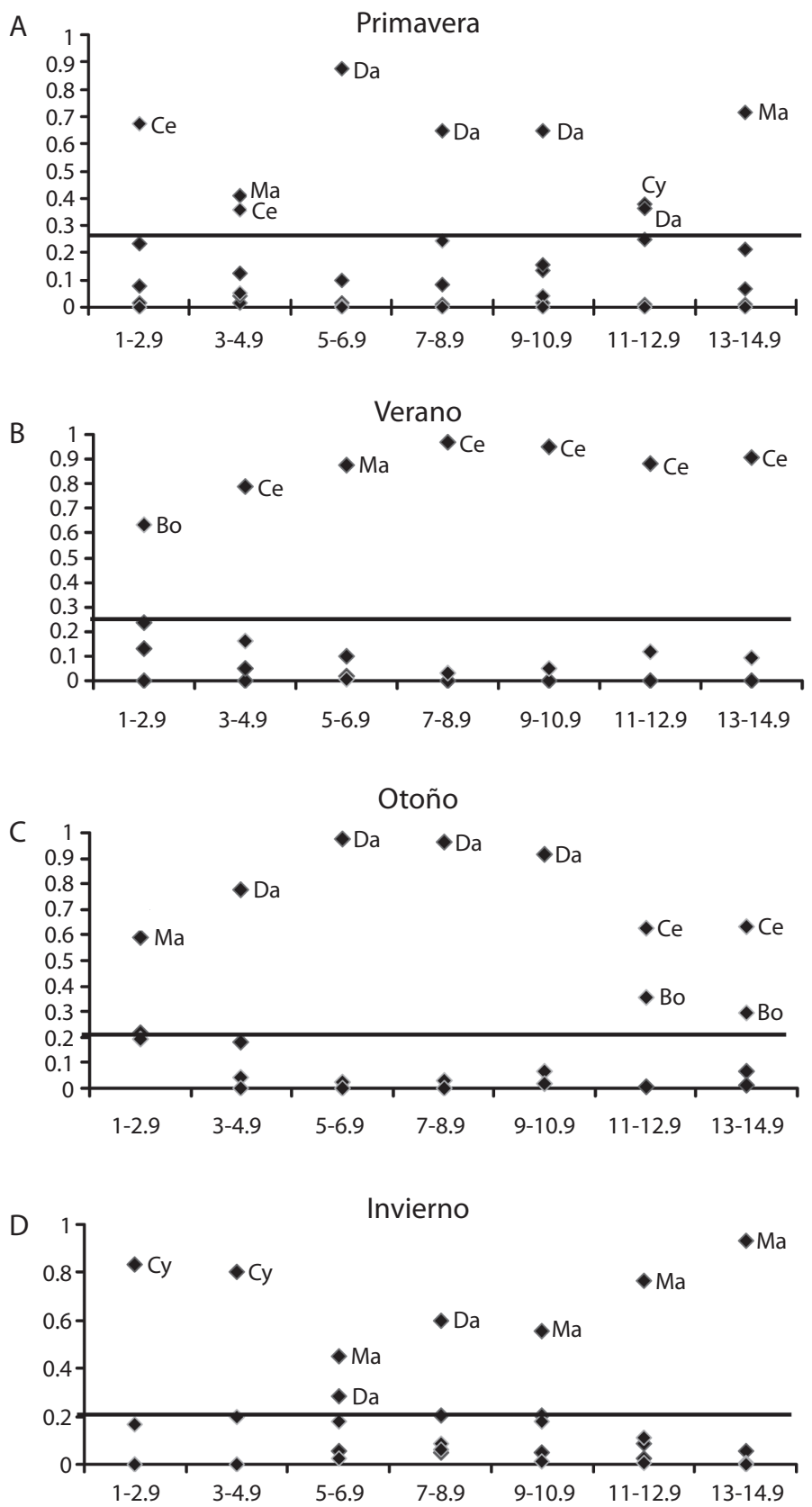

Fig. 2A. Coeficiente de selectividad de Menidia humboldtiana (primavera) en el embalse Tiacaque. Fig. 2B. Coeficiente de selectividad de Menidia humboldtiana (verano) en el embalse Tiacaque. Fig. 2C. Coeficiente de selectividad de Menidia humboldtiana (otoño) en el embalse Tiacaque. Fig. 2D. Coeficiente de selectividad de Menidia humboldtiana (invierno) en el embalse Tiacaque.

Fig. 2A. Selectivity coefficient of Menidia humboldtiana (spring) in the Tiacaque reservoir. Fig. 2B. Selectivity coefficient of Menidia humboldtiana (summer) in the Tiacaque reservoir. Fig. 2C. Selectivity coefficient of Menidia humboldtiana (autumn) in the Tiacaque reservoir. Fig. 2D. Selectivity coefficient of Menidia humboldtiana (winter) in the Tiacaque reservoir. 
$\mathrm{cm}$

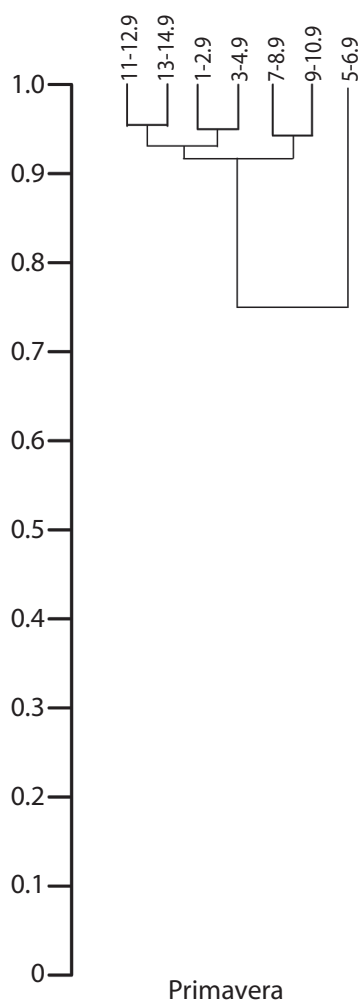

$\mathrm{cm}$

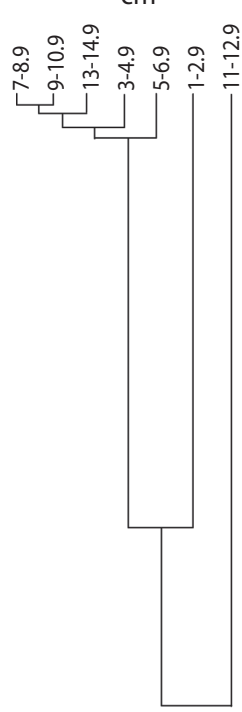

$\mathrm{cm}$

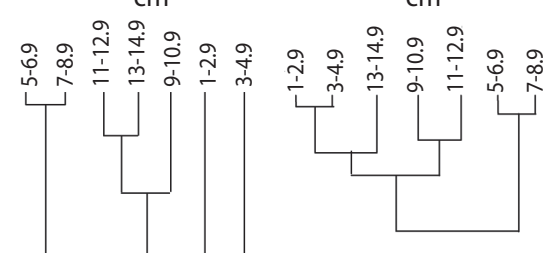

Fig. 3. Solapamiento alimentario entre los intervalos de longitud de Menidia humboldtiana en el embalse Tiacaque.

Fig. 3. Feeding overlap between the sizes of Menidia humboldtiana in the Tiacaque reservoir.

y otoño, en esta última época compartió los contenidos con Bosmina, a pesar de que este género registró su menor abundancia (7.2\%) durante todo el periodo de estudio, fue el alimento principal en la dieta en invierno, esto se puede explicar si consideramos que este género es depredado selectivamente por esta especie, coincidiendo con lo reportado por Sánchez et al. (2009); por su parte Ceriodaphnia fue más consumida en el verano.

En los peces de 7-8.9cm Daphnia contribuyó con buenos porcentajes a la dieta en primavera y otoño, compartiendo los contenidos estomacales con Cyclops y Bosmina respectivamente, en el verano disminuyó a dos el número de géneros ingeridos, siendo Ceriodaphnia el más importante aportando el 95.5\% a la dieta. Cyclops y Daphnia fueron los principales componentes en la dieta de los peces de $1-4.9 \mathrm{~cm}$ y de $9-14.9 \mathrm{~cm}$ en primavera, Cyclops y Bosmina (este último aportó con el $54.28 \%$ del zooplancton) en invierno, Ceriodaphnia en el verano y Ceriodaphnia y Bosmina en otoño. Las tallas más grandes de $11-14.9 \mathrm{~cm}$ de longitud consumieron principalmente a Cyclops en primavera coincidiendo con su máxima abundancia (56.99\% del zooplancton) e invierno. Para Ceriodaphnia en verano y otoño, ambos porcentajes en el ambiente fueron altos (27.70-40.01\%).

M. humboldtiana consumió de manera constante a Bosmina, Ceriodaphnia, Daphnia Cyclops y Mastigodiaptomus por los intervalos de talla de $5-14.9 \mathrm{~cm}$, la diferencia radicó en las proporciones de éstos en los contenidos estomacales. Así podemos mencionar que 
Moina se vio consumida por todas las tallas, pero resultó más importante en los estómagos (11.18-28.20\%) de los peces más grandes (11$14.9 \mathrm{~cm})$. A diferencia, Keratella fue para las tallas de $5-12.9 \mathrm{~cm}$ y Alona sólo para las tallas de $3-4.9 \mathrm{~cm}$; es importante mencionar que la contribución de estos tres últimos géneros en los contenidos estomacales fue mínima.

Cyclops fue el alimento más importante para las tallas pequeñas y grandes $(1-4.9 \mathrm{~cm}$ y 9-14.9cm respectivamente) y Daphnia para los peces de $5-10.9 \mathrm{~cm}$ de longitud. Esta alimentación diferencial en función de la talla se puede considerar como una estrategia para disminuir la competencia intraespecífica (Krebs 1985). Este mismo comportamiento fue observado por Navarrete \& Cházaro (1992) y Sánchez et al. (2009).

Los géneros Ceriodaphnia, Daphnia, Bosmina, Cyclops y Mastigodiaptomus, fueron seleccionados activamente por M. humboldtiana. Sánchez et al. (2009) reportan para esta especie una selectividad sólo por dos géneros (Daphnia y Bosmina) en el embalse Danxhó. Esta diferencia en la selectividad puede explicarse en primera instancia a la mayor abundancia de los mismos en el ambiente, manifestándose una selectividad por varios grupos alimenticios al aumentar la densidad (Werner \& Hall 1974). Otro aspecto que explica este comportamiento es la ausencia en Tiacaque de especies competidoras que utilicen el mismo recurso, a diferencia en Danxhó en donde sí existen dichas especies como Algansea tincella, Cyprinus carpio y Carassius auratus (Navarrete \& Sánchez 1991), que utilizan el mismo recurso en alguna etapa de su desarrollo.

Ceriodaphnia, Daphnia y Mastigodiaptomus fueron seleccionados por seis de los siete intervalos de talla (excepto por los peces de $5-6.9 \mathrm{~cm}, 1-2.9 \mathrm{~cm}$ y $7-8.9 \mathrm{~cm}$ respectivamente), al respecto estos tres géneros corresponden al zooplancton de talla grande, lo que propicia que los peces detecten con mayor facilidad a estos organismos, manifestándose una selectividad por tallas (Werner \& Hall 1974, O’Brien 1979, Clements \& Livingston 1984). A diferencia los géneros del zooplancton de talla pequeña
Cyclops y Bosmina sólo se vieron seleccionados por los peces pequeños $(1-4.9 \mathrm{~cm})$ y grandes $(11-14.9 \mathrm{~cm})$. Hay que recordar que la selectividad por algún tipo de alimento implica la relación de los porcentajes de los contenidos estomacales con su contraparte en el ambiente.

En relación al solapamiento de las tallas del pez blanco, se obtuvieron valores elevados de solapamiento por todos los intervalos de longitud (excepto las tallas pequeñas de $1-2.9 \mathrm{~cm}$ y $11-12.9 \mathrm{~cm}$ en verano) en las cuatro épocas del año, lo que indicó una fuerte competencia intraespecífica por la utilización del recurso alimenticio. Este comportamiento fue un poco diferente al reportado para esta especie en el embalse Danxhó (Sánchez et al. 2009), en donde se reportaron valores elevados de solapamiento pero no por todos los intervalos de longitud ni en todas las épocas del año.

En importante considerar que las mayores abundancias de los grupos alimenticios en el ambiente, la selectividad de los peces por más tipos de alimento y la ausencia de especies de peces competidoras en el embalse Tiacaque propiciaron las diferencias encontradas respecto al embalse Danxhó.

Menidia humboldtiana es una especie zooplanctófaga, que realiza una depredación selectiva por los géneros Ceriodaphnia, Daphnia, Bosmina, Cyclops y Mastigodiaptomus y presenta un elevado solapamiento trófico entre sus diferentes intervalos de longitud.

\section{RESUMEN}

Menidia humboldtiana es una especie nativa muy apreciada por su delicado sabor. Se determinó el espectro trófico, selectividad y solapamiento trófico de ésta, durante 1995 (épocas del año), se obtuvieron muestras de zooplancton e identificaron a nivel genérico. Los peces capturados se agruparon en intervalos de longitud estándar para cada época. Se analizaron los contenidos estomacales (método volumétrico, Laevastu), selectividad (Chesson) y solapamiento trófico (Morisita). Se registraron 14 géneros de zooplancton; Bosmina el más abundante (29 625ind/10L) seguido por Cyclops (9 496ind/10L) ambos en primavera. Los peces pequeños $(1-4.9 \mathrm{~cm})$ consumen a Cyclops en altos porcentajes en primavera e invierno, 61.24-69.82\% respectivamente. Ceriodaphnia es consumida por peces de $3-10.9 \mathrm{~cm}$ y de $13-14.9 \mathrm{~cm}$ con $72.41-95.5 \%$ en verano; en 
otoño las tallas pequeñas ingieren a Mastigodiaptomus y Ceriodaphnia; Daphnia y Bosmina por peces de 5-8.9cm y los más grandes $(9-14.9 \mathrm{~cm})$ a Ceriodaphnia. M. humboldtiana realiza una depredación selectiva por Ceriodaphnia, Daphnia, Mastigodiaptomus, Bosmina y Cyclops. El solapamiento trófico fue muy marcado entre todas las tallas en primavera, otoño e invierno, a diferencia en verano los peces de $1-2.9$ y $11-12.9 \mathrm{~cm}$ no registraron un solapamiento con otros intervalos de longitud. M. humboldtiana es una especie zooplanctófaga, que realiza una depredación selectiva y un marcado solapamiento trófico entre los intervalos de longitud.

Palabras clave: selectividad alimenticia, Menidia humboldtiana, solapamiento trófico, pez blanco, zooplancton.

\section{REFERENCIAS}

Arcifa, M.S., T.G. Norticote \& O. Froehlich. 1991. Interactive ecology of two cohabiting characin fisher (Astyanax fasciatus and Astyanax bimaculatus) in a eutrophic Brazilian reservoir. J. Trop. Ecol. 7: 257-268.

Brooks, J.L. \& S.I. Dodson. 1965. Predation body size and competition of plankton. Science 150: 28-35.

Chesson, J. 1978. Measuring preference in selective predation. Ecology 59: 211-215.

Clements, H.W. \& R.J. Livingston. 1984. Prey selectivity of the fringed filefish Monacanthus ciliatus (Pisces: Monacanthidae): role of prey accessibility. Mar. Ecol. Prog. Ser. 16: 291-295.

Dussart, B. \& D. Defaye. 1995. Guides to the identification of the microinvertebrates of the continental waters of the World: Introduction to the Copepoda. Academic, Londres, Inglaterra.

Edmonson, W.T. 1959. Freshwater biology. Wiley, Nueva York, Nueva York, EEUU.

Elias, F.G., N.A. Navarrete \& J.L. Rodríguez. 2008. Alimentación de Chirostoma humboldtianum (Valenciennes); (Pisces: Atherinopsidae) en el estanque JC en Soyaniquilpan, Estado de México. Rev. Chapingo 14: $129-134$.

García, E. 1973. Modificación del sistema de clasificación climático de Köppen (para adaptarlo a las condiciones de la República Mexicana). Instituto de Geografía, Universidad Nacional Autónoma de México, México D.F., México.
Gaviño, G., J.C. Juárez \& H.H. Figueroa. 1982. Técnicas biológicas selectas de laboratorio y de campo. Limusa, México D.F., México.

Krebs, M.E. 1985. Ecología estudio sobre la distribución y abundancia. Harla, México D.F., México.

Laevastu, T. 1971. Manual de métodos de biología pesquera. Acribia, Zaragoza, España.

Miller, R.R. 2005. Freshwater fishes of Mexico. University of Chicago, Chicago, EEUU.

Navarrete, S.N., J. Rubio, M. Domínguez \& E. Fernández. 2007. Espectro trófico y trama trófica de la ictiofauna del embalse San Miguel Arco en Soyaniquilpan, Estado de México. Rev. Zool. 18: 1-12.

Navarrete, S.N. \& R. Sánchez. 1991. Variaciones alimenticias de los peces del embalse Danxhó, en tres épocas del año. II Congreso Nacional de Ictiología. Monterrey, Nuevo León, México.

Navarrete, S.N. \& S. Cházaro. 1992. Espectro trófico del charal Chirostoma humboldtianum del embalse San Felipe Tiacaque, Estado de México. Rev. Zool. 3: 28-34.

O'Brien, W.L. 1979. The predator prey interaction of planktivorous fish and zooplankton. Am. Scien. 67: 572-581.

Pennak, R.W. 1989. Freshwater invertebrates of the United States. Wiley, Nueva York, Nueva York, EEUU.

Sánchez, M.R., M.Y. Fermín \& A. Mendoza. 2009. Selectividad del zooplancton y solapamiento trófico entre tallas del pez Menidia humboldtiana (Atheriniformes: Atherinopsidae) en el embalse Danxhó, México. Rev. Biol. Trop. 57: 187-194.

Vanderploeg, H.A. \& D. Scavia. 1979. Two selectivity indices for feeding with special reference to zooplankton grazing. J. Fish. Res. Board Can. 36: 362-365.

Wells, L. 1970. Effects of alewife predation on zooplankton populations in lake Michigan. Limnology 15: 556-565.

Werner, E.E. \& D.J. Hall. 1974. Optimal foraging and the size selection of prey by the bluegill sunfish (Lepomis macrochirus). Ecology 55: 1042-1052.

Yubi, A.M.A., N.A. Navarrete, G. Elías, G. Vázquez \& E.S. Urrieta. 2008. Relaciones tróficas de los peces del embalse San Miguel Arco, de Soyaniquilpan, México. Rev. Chapingo 14: 33-38. 